EPJ Web of Conferences 24, 07005 (2012)

DOI: $10.1051 /$ epjconf/20122407005

(C) Owned by the authors, published by EDP Sciences - SIF, 2012

\title{
Quantification of uranium-238 in environmental samples using gamma-ray spectrometry
}

\author{
M. Hult*, E. Andreotti, R. GonzÁlez de Orduña, \\ S. Pommé and E. Yeltepe
}

EC-JRC Institute for Reference Materials and Measurements Retieseweg 111, B-2440 Geel, Belgium

\begin{abstract}
A large number of environmental samples are routinely measured world-wide using gamma-ray spectrometry, some of its assets being easy sample preparation and comprehensive data for many radionuclides in one analysis. Although other techniques can be considered more suitable for analysing ${ }^{238} \mathrm{U}$ in environmental samples, it is also routinely done by gamma-ray spectrometry. One mainly uses $\gamma$-ray emissions following the decay of the first daughter, ${ }^{234} \mathrm{Th}$, for determining the ${ }^{238} \mathrm{U}$ activity. However, the low-energy gamma-rays at $63 \mathrm{keV}$ and $92.5 \mathrm{keV}$ are very difficult to quantify in a robust way due to high attenuation and interferences. This paper quantifies parameters affecting the possibility of making robust quantification of ${ }^{238} \mathrm{U}$ via ${ }^{234} \mathrm{Th}$ using gamma-ray spectrometry. It addresses the use of correct decay data, suitable detectors, optimised sample size, enhanced spectral amplification, correction for peak interferences and control of background.
\end{abstract}

\section{Introduction}

Gamma-ray spectrometry using HPGe-detectors is a widely used technique in laboratories monitoring environmental radioactivity. The possibility to

${ }^{*}$ E-mail: mikael.hult@ec. europa.eu

This is an Open Access article distributed under the terms of the Creative Commons Attribution License 2.0, which permits unrestricted use, distribution, and reproduction in any medium, provided the original work is properly cited. 
quantify the activity of several radionuclides in one single analysis makes it an efficient analytical tool. However, the detection limits for various radionuclides vary greatly depending on the matrix in which they are. The detection limits of radionuclides emitting gamma-rays of low energy $(<100 \mathrm{keV})$ suffer from being in a matrix with other radionuclides emitting gamma-rays of higher energy due to interferences with X-rays and scattered gamma-rays. This is generally the case when quantifying ${ }^{238} \mathrm{U}$ in an environmental sample like soil.

Uranium-238 is a primordial radionuclide that is important to monitor. It decays by alpha-decay to ${ }^{234} \mathrm{Th}$, which generates low-energy gamma-rays with very low emission probability. It can be measured with low detection limits using alpha-particle spectrometry and mass spectrometry. However, in order to save time on sample preparation and analysis, it is very common to measure ${ }^{238} \mathrm{U}$ using gamma-ray spectrometry [1]. The $\gamma$-ray transitions in ${ }^{234} \mathrm{Th}$ (following the decay of ${ }^{238} \mathrm{U}$ ) are too weak for this purpose. Instead the gamma-rays following the $\beta$-decays of ${ }^{234} \mathrm{Th}\left(T_{1 / 2}=24.1 \mathrm{~d}\right)$ and its daughter ${ }^{234} \mathrm{~Pa}^{\mathrm{m}}\left(T_{1 / 2}=1.17 \mathrm{~m}\right)$ are used for identification and quantification of ${ }^{238} \mathrm{U}$. Care must of course be taken to ensure that secular equilibrium exists between ${ }^{234} \mathrm{Th}$ and ${ }^{238} \mathrm{U}^{1}$.

In pure uranium samples the detection limits (in $\mathrm{mBq}$ ) are low and the following gamma-rays can be used: $63 \mathrm{keV}$ (doublet), $92.5 \mathrm{keV}$ (doublet), $767 \mathrm{keV}$ and $1001 \mathrm{keV}$. The gamma-rays at 767 and $1001 \mathrm{keV}$ are robust for quantification but due to their low emission probabilities $(0.317 \%$ and $0.842 \%$, respectively) they are often swamped by the background from other radionuclides in the environmental sample itself. It is thus necessary to use the two low-energy gamma-lines. This introduces several problems that are outlined and quantified in this study, which was initiated through participation in the IAEA-CRP "Benchmarking Calibration for Low-Level Gamma Spectrometric Measurements of Environmental Samples". The first problem is that the four low-energy peaks appear as two doublets in a "normal" HPGe-detector spectrum. In this paper we will simply refer to the two doublet peaks as if they were singlet peaks at $63 \mathrm{keV}$ and $92.5 \mathrm{keV}$.

\footnotetext{
${ }^{1}$ In case it can be proven that equilibrium between ${ }^{238} \mathrm{U}$ and ${ }^{226} \mathrm{Ra}$ exist (e.g. in certain geological samples), the latter can be used as a measure of the ${ }^{238} \mathrm{U}$ activity. Furthermore, if one knows that there is a natural isotopic abundance of uranium isotopes in the sample and ${ }^{235} \mathrm{U}$ can be quantified, this provides an alternative way to calculate the ${ }^{238} \mathrm{U}$ activity without having to consider the radioactive equilibrium.
} 


\section{Analytical considerations}

\subsection{Use of correct decay data}

Numerous measurements of the $\gamma$-ray intensities and energies following the decay of ${ }^{234} \mathrm{Th}$ to ${ }^{234} \mathrm{~Pa}$ have been performed, and best values were derived from evaluations of the available data sets (see $[2,3]$ and references therein). Apparently the emission probabilities vary significantly from one source to another. For example, for the most intense $\gamma$-ray at $63.290 \mathrm{keV}$ one finds in reference work absolute intensities of $3.75(8) \%^{2}$ [2], $3.69(7) \%$ [3], $4.8 \%$ [4], 4.1 (7)\% [5], 4.8 (6)\% [6], 4.00\% [7], $3.7(2) \%$ [8], 3.6 (1)\% [9], $4.1(7) \%$ [10], $3.7(4) \%$ [11]. Recent evaluations $[2,3]$ have to be preferred, as they must have gained accuracy due to the recent measurement of the main peak intensity by Abousahl et al. [12]. The intensities of the other gamma-rays were derived from relative intensity measurements by Godart and Gizon [13] in review [3], while review [2] relied on Chu and ScharffGoldhaber [14], mainly because in this case the U KX-rays contributions were resolved from the gamma-ray peaks situated in the (90-115) keV energy region. In this paper we use $I_{\gamma}=3.75$ (8)\% [2] for the $63.29 \mathrm{keV}$ emission probability, although it is very common to find recent articles using much higher values, up to $4.8 \%$. The review [2] yields furthermore $I_{\gamma}(62.88 \mathrm{keV})=$ $0.016(4) \%, I_{\gamma}(92.38 \mathrm{keV})=2.18(19) \%, I_{\gamma}(92.80 \mathrm{keV})=2.15(19) \%$, and $I_{\gamma}(112.81 \mathrm{keV})=2.15(22) \%$ as most relevant additional peaks.

\section{$2.2 \quad$ Use of a suitable detector}

It is well-known in some fields of radionuclide analysis, like e.g. safeguards and nuclear fuel studies, that low energy gamma-ray emitters like ${ }^{234} \mathrm{Th}$, ${ }^{238-242} \mathrm{Pu}$ and ${ }^{241} \mathrm{Am}$, are best measured using a thin HPGe-detector with a thickness of only a few mm. However, when measuring ${ }^{234} \mathrm{Th}$ in environmental samples, one often has to make use of available instrumentations not optimised for the purpose of measuring gamma-rays below $100 \mathrm{keV}$ or to compromise with the detection of other radionuclides emitting gammarays with higher energies. In recent years the sales of large volume p-type HPGe-detectors with thin upper deadlayer have increased. Such detectors provide high detection efficiency for gamma-ray energies over a wide range, which to some extent has taken over measurements previously performed with small n-type detectors.

\footnotetext{
${ }^{2}$ Calculated from intensity of doublet $=3.72 \%$ and divided by $(100+0.75) \%$.
} 


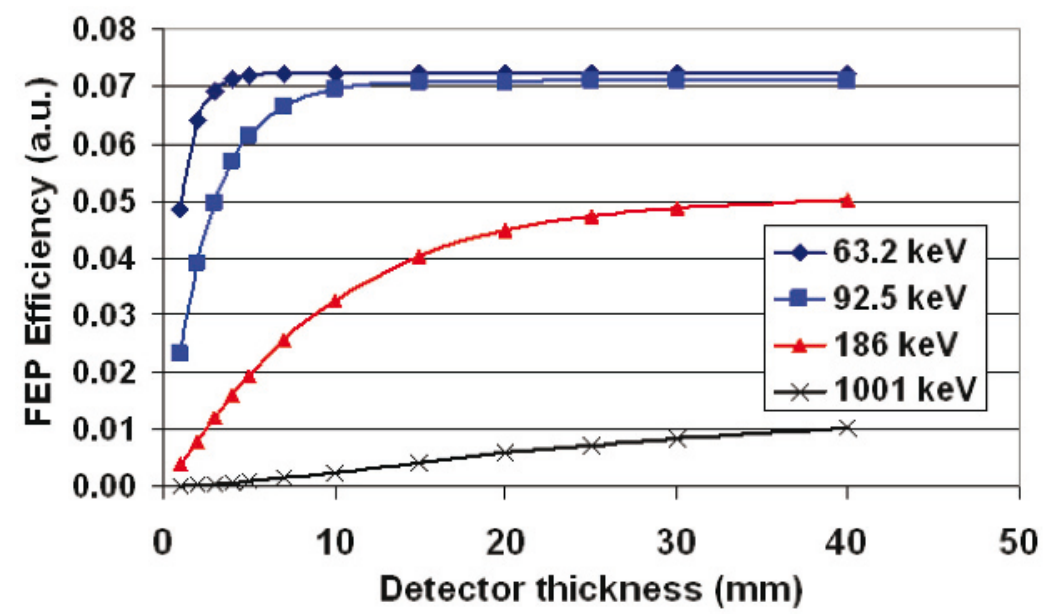

Figure 1: Monte Carlo calculations of the FEP efficiency as a function of the detector thickness for a planar crystal with a radius of $50 \mathrm{~mm}$ and a top deadlayer thickness of $0.5 \mu \mathrm{m}$. The sample was a $40 \mathrm{~mm}$ high cylinder of dried soil with density $1.3 \mathrm{~g} / \mathrm{cm}^{3}$, located directly on the endcap.

Two main advantages using "thin" HPGe-detectors for measurement of low energy gamma-rays are: (i) The low energy gamma-rays do not penetrate very deep. Figure 1 shows that for the $63 \mathrm{keV}$ line, already with a detector thickness of $3 \mathrm{~mm}$, the full-energy peak (FEP) efficiency has reached $96 \%$ of the FEP efficiency of a $30 \mathrm{~mm}$ thick detector. The rear part of the Ge-crystal, in this case, has no added value since it mainly increases the background at the lower energies. A Compton suppression system will help only to some modest extent, since its effect below $100 \mathrm{keV}$ is limited unless a complicated arrangement with an extra detector "behind" the Ge-crystal is used [15]. (ii) The resolution of a HPGe-detector degrades with increased size and particularly at low energies a high resolution is needed to resolve the many $\gamma$-rays, X-rays, backscatter and escape peaks lying close in energy.

The use of thin deadlayer detectors improves the robustness of quantification of low-energy gamma-rays since the slope of the FEP efficiency curve is much less steep compared to a detector with thick deadlayer (see figs. 2 and 3). Furthermore, the extent and effect of a deadlayer is difficult to assess accurately since it depends on the electrical field, which depends on doping profile and implantation/diffusion depth, and this can vary along a crystal axis or height. Therefore having a thin deadlayer reduces the impact of such changes as was shown by Johnston et al. [16]. 


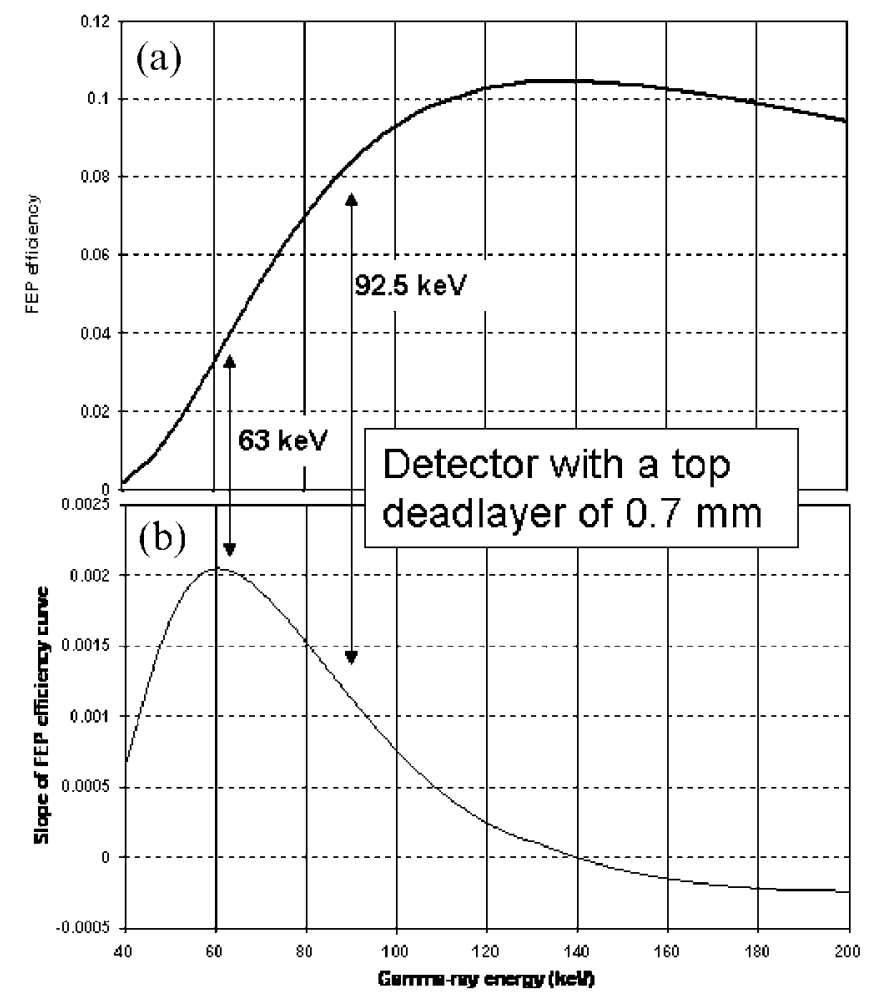

Figure 2: (a) The FEP efficiency for a detector with a thick $(0.7 \mathrm{~mm})$ deadlayer and (b) the derivative of the curve in (a). The sample was dried soil with the following atomic composition: $\mathrm{H}(6), \mathrm{C}(5), \mathrm{O}(4), \mathrm{Al}(4), \mathrm{Si}(5)$ and $\mathrm{Fe}(1)$ and $1.5 \mathrm{~g} / \mathrm{cm}^{3}$ density. The sample was placed in a Teflon container with $0.2 \mathrm{~cm}$ bottom thickness and $5 \mathrm{~cm}$ internal radius. The height of the sample was $1 \mathrm{~cm}$. The Teflon container was centered directly on the endcap.

\subsection{Optimising the sample}

Figure 4 shows the count rate of the FEP as a function of the sample thickness for different gamma-rays from a soil sample with a density of $1.3 \mathrm{~g} / \mathrm{cm}^{3}$ in a cylindrical container with an inner diameter of $50 \mathrm{~mm}$. Also the continuum background from higher energy gamma-rays increases with sample size. However, for a "low-Z sample" with relatively low density, say around $2 \mathrm{~g} / \mathrm{cm}^{3}$, it is possible to use relatively thick samples $(50-100 \mathrm{~mm})$ as the count rate of the $63 \mathrm{keV}$ and $92.5 \mathrm{keV}$ peaks might increase more than the square root of the background. It is important to know the concentration of heavy elements at percent level since they will influence the self-attenuation at low energies strongly. 


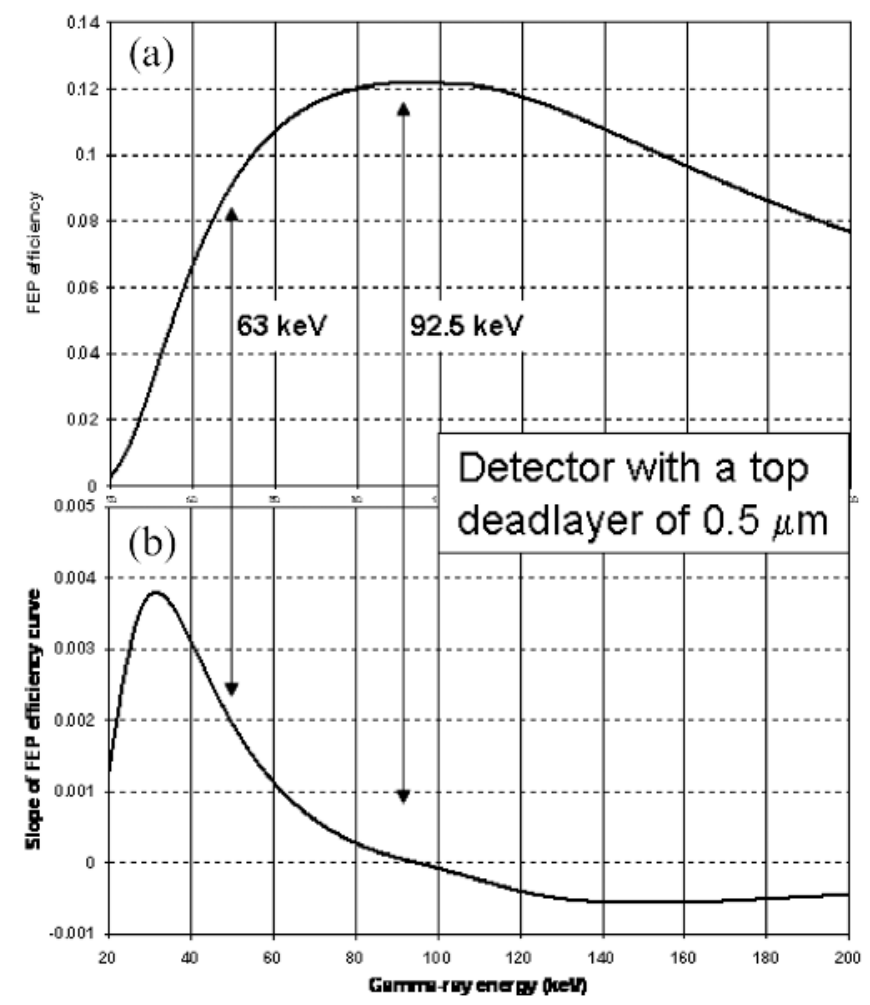

Figure 3: (a) The FEP efficiency for a detector with a thin $(0.5 \mu \mathrm{m})$ deadlayer and (b) the derivative of the curve in (a). The same sample as for figure 2.

\subsection{Use of suitable amplification}

When measuring with an HPGe-detector that is not very small, the amplification is often made such that one can detect the $2614 \mathrm{keV}$ gammaray from ${ }^{208} \mathrm{Tl}$. Using 8192 channels this results in a bin-width of about $0.33 \mathrm{keV}$. Such an amplification is not optimal for measuring gamma-rays below $100 \mathrm{keV}$, where the resolution (FWHM) of the detector can be $0.5 \mathrm{keV}$. In case of poop counting statistics, this can result in "peaks" of one or two channels. Such peaks are not suitable to fit with any gamma-ray spectrometry software. Furthermore the potential non-linearities of the energycalibration that are known to be greater at low energies will have a greater impact when a peak covers fewer channels. A more suitable amplification is to use e.g. the $662 \mathrm{keV}$ line from ${ }^{137} \mathrm{Cs}$ as an upper limit near to channel 8192 , giving a bin-width of $0.08 \mathrm{keV}$. Then a peak with a full-width-at-halfmaximum of $0.5 \mathrm{keV}$ (FWHM) would be covered by roughly ten channels. 


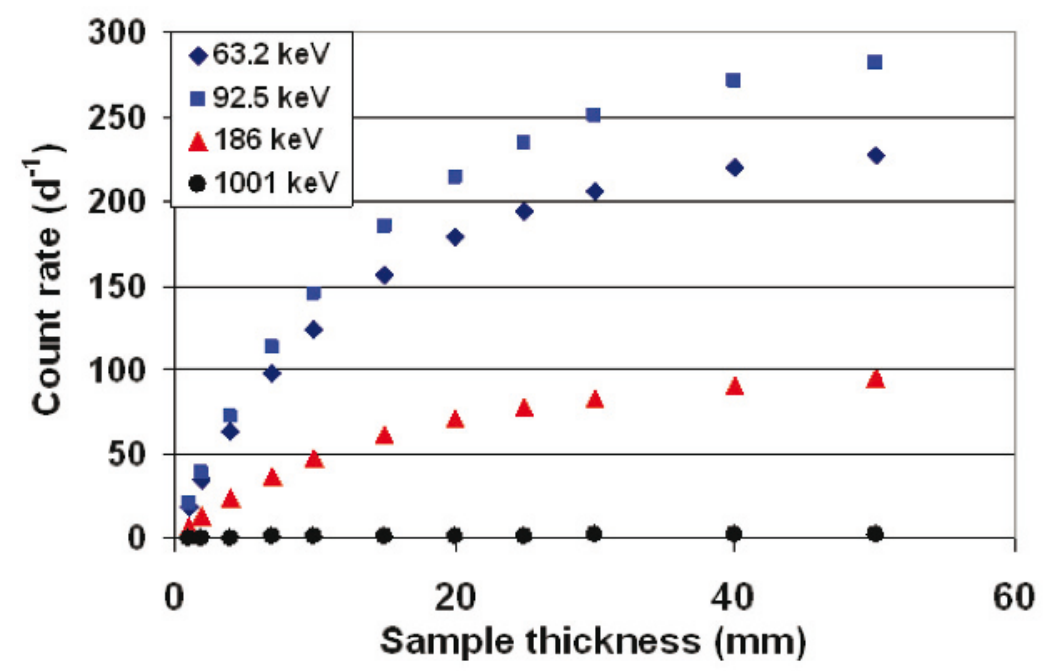

Figure 4: The count rate of four gamma lines for a fixed detector from a sample with density $1.3 \mathrm{~g} / \mathrm{cm}^{3}$ as a function of the sample thickness. The assumed massic activity was $10 \mathrm{mBq} / \mathrm{g}$ of ${ }^{238} \mathrm{U}$ and ${ }^{226} \mathrm{Ra}$.

The bin-width must also be considered when performing Monte Carlo simulations. At low energies 8 important detector response features need to be resolved in a narrow energy range (within $53 \mathrm{keV}$ in the case of the $63 \mathrm{keV}$ peak). Starting from the highest energy and going down, those features are: (i) the FEP, (ii) single low angle Compton scattering in the sample generating photons that can end up in the FEP, (iii+iv) the $\mathrm{Ge}_{\alpha}$ and $\mathrm{K}_{\beta}$ escape peaks, (v) the backscatter peak, (vi) the Compton edge (note that it is at lower energy than the backscatter peak), (vii+viii) $\mathrm{Ge}_{\alpha}$ and $\mathrm{K}_{\beta}$ peaks.

\subsection{Subtraction of interfering peaks}

Depending on the sample composition, there are many X-rays and gammarays that can interfere with the 63 and $92.5 \mathrm{keV}$ lines. Since gamma-ray spectrometry of ${ }^{238} \mathrm{U}$ is a very common activity, there are numerous papers (see e.g. [17-22]) dealing with this and listing interfering peaks and ways to resolve this. For robust quantification of ${ }^{238} \mathrm{U}$ it is important that the interfering peaks are not too dominating. The main interference for the $92.5 \mathrm{keV}$ doublet of ${ }^{234} \mathrm{Th}$ is the thorium X-ray $\mathrm{K}_{\alpha 1}(93.351 \mathrm{keV})$. As for the gamma-rays, the values available in literature for the intensities of $\mathrm{Th}$ $\mathrm{X}$-rays present significant differences. The values used in this article are from the DDEP-website [2]. In environmental samples, thorium X-rays can 
be originated from the decay of: ${ }^{228} \mathrm{Ac}(4.1(11) \%),{ }^{235} \mathrm{U}(5.76(14) \%)$ and ${ }^{238} \mathrm{U}(0.0018(5) \%)$. Considering the low emission probability of ${ }^{238} \mathrm{U}$ and a nominal ${ }^{235} \mathrm{U} /{ }^{238} \mathrm{U}$ activity ratio (0.046) the main interference come from ${ }^{228} \mathrm{Ac} \mathrm{Th} \mathrm{K}_{\alpha 1}$. If the activity ratio ${ }^{238} \mathrm{U}\left({ }^{234} \mathrm{Th}\right) /{ }^{228} \mathrm{Ra}\left({ }^{228} \mathrm{Ac}\right)$ equals 0.95 in a sample, the count rate from the $92.5 \mathrm{keV}$ doublet and the $93.3 \mathrm{X}$-ray are equal. Activity ratios near to 1 are often encountered in environmental samples and results thus in almost equally sized peaks.

\subsection{Use of suitable stable reference materials}

It is very important to calibrate measurements using good quality reference materials of a geometry and matrix that is similar to the samples that are being measured. Most reference materials do, however, suffer to a greater or lesser degree from aging due to chemical reactions, diffusion and evaporation. There are also known cases where reference materials have inhomogeneous distribution of ${ }^{238} \mathrm{U}$, which can occasionally be localised in hot-spots in e.g. soil samples. It is therefore important not to be too reliant on one reference material. Furthermore in the case of the 63 and $92.5 \mathrm{keV}$ peaks, there is very much influence of the sample composition on the self-absorption and interference corrections. Therefore it is dangerous to blindly use the detection efficiency obtained from a single reference sample.

An important consideration is to look at how a reference value was determined. For many primordial radionuclides in environmental samples it is not so easy with spiking of primary standards and reference values may have been determined as mean values from intercomparisons of non-expert laboratories, where systematic errors may have been hidden.

\subsection{Calculating efficiency transfer and absolute efficiency}

When calculating efficiency using Monte Carlo codes one needs to know geometrical details of the detector and the sample. Low energy gammarays are more sensitive to errors in these geometrical factors. Gasparro et al. [23] showed that failing to include e.g. the rounded edges of a crystal in a computer simulation, can alter the calculated efficiency of several tens of percent for energies below $100 \mathrm{keV}$. The other most crucial parameters are: Sample composition, container bottom thickness, detector window thickness, distance from crystal to window, deadlayer thicknesses (side and top). For low-energy gamma-rays it is common that calculated FEP efficiencies can be off with up to $100 \%$. Therefore, it is strongly recommended to use efficiency transfer calculations as errors in the computer model more or less cancel 
out. Furthermore, Vidmar et al. [24] showed that different Monte Carlo codes generate the same results when calculating efficiency transfer factors.

\subsection{Background}

Perhaps the most obvious parameter for performing robust measurements is a good control of the background. There is a strong trend in placing HPGe-detectors underground [25], meaning at a depth of at least $10 \mathrm{~m}$ water equivalent [26]. It is clear that by reducing the cosmic ray background one can reach lower detection limits. However, when the cosmic ray continuum reduces, the peaks from radionuclides in the background appear. It is thus easier to see if the background changes. Performing measurements with low-background is thus important not only for activities in the $\mathrm{mBq}$ range but also for activities in the range of a few hundreds of $\mathrm{mBq}$ to some Bq. These are activities frequently encountered in monitoring laboratories. Furthermore, having a lower background means that one can measure smaller samples, which has many practical advantages with regards to sampling and storage.

The lowest detection limits for ${ }^{238} \mathrm{U}$ using gamma-ray spectrometry is obtained in small swipe samples containing only uranium, measured in the underground laboratory HADES (225 $\mathrm{m}$ deep) [27]. The detection limit derived from that measurement is $0.1 \mathrm{mBq}$ corresponding to $10 \mathrm{ng} \mathrm{U}$.

\section{Conclusions}

In order to improve robustness in determination of ${ }^{238} \mathrm{U}$ using gamma-ray spectrometry we can identify a few steps that may not be unrealistic to introduce although they all involve investments and spending longer time on each sample.

(i) Use of underground laboratory to reduce background in order to have better control of the background and reduce detection limits and robustness.

(ii) Use of two separate HPGe-detectors optimised for determination of low energy and high energy gamma-rays, or alternatively.

(iii) to make use of one large volume p-type detector with thin deadlayer with two chains of electronic with high amplification for low energies and low amplification for high energy gamma-rays.

It is important to use correct decay data and being aware that the peaks interfering with the $63 \mathrm{keV}$ and $92.5 \mathrm{keV}$ gamma-rays vary depending on the sample matrix. 
The problems with quantifying ${ }^{238} \mathrm{U}$ discussed in this paper are of course more severe at even lower energies like the $59.5 \mathrm{keV}$ from ${ }^{241} \mathrm{Am}$ and $46.5 \mathrm{keV}$ from ${ }^{210} \mathrm{~Pb}$. This was manifested e.g. in a recent IAEA intercomparison on ${ }^{210} \mathrm{~Pb}$ in soil [28]. All in all, one needs to exercise great care when analysing data below $100 \mathrm{keV}$ and investigate any automatic peak fitting and interference correction carefully. Note also the abundant uncertain decay data in use in different laboratories. The discrepant decay data is to some extent a consequence of all the other sources of errors at low energies mentioned in this paper.

\section{Acknowledgements}

The work was carried out as part of the IAEA Research Agreement No. 15085 between the IAEA and EC-JRC-IRMM. Matthias Köhler (VKTARossendorf) and Werner Preuße (Staatliche Betriebsgesellschaft für Umwelt und Landwirtschaft, Chemnitz) are acknowledged for valuable input to this study.

\section{References}

[1] Saïdou, Bochud F., Laedermann J.P., Kwato Njock M.G. and Froidevaux P., Appl. Radiat. Isot., 66(2) (2008) 215-222.

[2] Luca A., Table de Radionucléides, ${ }^{234} \mathrm{Th}$, LNE-LNHB, CEA, 2009, http://www.nucleide.org/DDEP_WG/DDEPdata.htm (accessed 22 November 2010).

[3] Browne E. and Tuli J.K., in Nuclear Data Sheets for $A=234$, Nuclear Data Sheets, 108 (2007) 681-772.

[4] Laboratoire National Henri Becquerel, Mini Table de Radionucléides (EDP Sciences) 2007.

[5] Wahl W., $\alpha \beta \gamma$-Table, Radionuclide Handbook for Laboratory workers in Spectrometry, Radiation Protection and Medicine, version 4.3.2 (Isus Publications, Schliersee, Germany) 2010.

[6] Laboratoire National Henri Becquerel, Nuclèide 2000, version 30 June 2004.

[7] Magill J. et al., Nuclides 2000, An electronic chart of the nuclides, version 1.00, European Commission 1999. 
[8] Longworth G. et al., The Radiochemical Manual, ISBN 0-7058-1768-7 (AEA Technology plc, Harwell, Oxfordshire, OX11 0RA) 1998.

[9] Schoetzig U. and Schrader H., PTB-Bericht Ra-16/3, errata 1991 (PTB, Braunschweig) 1989.

[10] Schoetzig U. and Schrader H., PTB-Bericht Ra-16/5 (PTB, Braunschweig) 1998.

[11] National Nuclear Data Center (NNDC), Brookhaven National Laboratory, Upton, NY, http://www.nndc.bnl.gov/ (accessed 22 November 2010).

[12] Abousahl S., Van Belle P., Lynch B. and Ottmar H., Nucl. Instrum. Methods Phys. Res. A, 517 (2004) 211.

[13] Godart J. and Gazon A., Nucl. Phys. A, 217 (1973) 159-176.

[14] Chu Y.Y. and Scharff-Goldhaber G., Phys. Rev. C, 17 (1978) 15071509 .

[15] Wahl W. et al., Nucl. Instrum. Methods Phys. Res. A, 369 (1996) 627633.

[16] Johnston P.N., Hult M. and Gasparro J., Appl. Radiat. Isot., 64 (2006) 1323-1328.

[17] El-Daoushy F. and Hernandez F., Analyst, 127(7) (2002) 981-989.

[18] Papachristodoulou C.A., Assimakopoulos P.A., Patronis N.E. and Ioannides K.G., J. Environ. Radioact., 64(2-3) (2003) 195-203.

[19] Yücel H. et al., Appl. Radiat. Isot., 67(11) (2009) 2049-2056.

[20] Kaste J.M., Bostick B.C. and Heimsath A.M., Analyst, 131(6) (2006) 757-763.

[21] Huy N.Q. and Luyen T.V., Appl. Radiat. Isot., 61(6) (2004) 1419-1424.

[22] García-Talavera M., Appl. Radiat. Isot., 59(2-3) (2003) 165-173.

[23] Gasparro J., Hult M., Johnston P.N. and Tagziria H., Nucl. Instrum. Meth. A, 594 (2008) 196-201.

[24] Vidmar T. et al., Appl. Radiat. Isot., 68(12) (2010) 2352-2354. 
[25] Hult M., Metrologia, 44 (2007) S87-S94.

[26] Hult M., Gasparro J. and Preusse W., Acta Chimica Slovenica, 87 (2005) 1-6.

[27] Andreotti E. et al., in Proceedings from 3rd International Conference "Current Problems in Nuclear Physics and Atomic Energy" (Publishing Department of KINR, Kiev, Ukraine) 2011, pp. 601-605.

[28] Shakhashiro A. and Mabit L., Appl. Radiat. Isot., 67 (2009) 139-146. 